PART D

SUMMARY 
We have seen from the talks of A. B. Underhill and $R$. N. Thomas how the viewpoints of the many kinds of astronomers who came to this meeting differ, not only with respect to what they mean by "extended atmospheres" but also concerning methods for studying extended atmospheres.

For a long time spectroscopists and theoreticians of stellar atmospheres have not moved exactly in the same universe. This conference is an attempt to fill the gap, we all have seen that it is not easy, but progress has been made. I shall try to summarize the progress starting from the general conceptual approach and trying to come back to the particular cases that puzzle observers.

\section{THE CONCEPTS}

The problem, as I said, is that for a long time it was found to be very difficult to build models of the good classical type, that is in $\mathrm{RE}$ (radiative equilibrium), HE (hydrostatic equilibrium), LTE (local thermodynamic equilibrium), which might represent the most honest stars that theoreticians might be tempted to like. Most of the theoreticians were sufficiently aware of this fact not to give more than very qualitative suggestions on how to explain monsters. On the other hand, however, observers, i.e., spectroscopists, have had a tendency to concentrate on stranger and stranger objects, to have more fun, probably, and they seemed to have been satisfied with zero-order-approximation interpretations--often being so crude as to assume LTE! In a way they were like the entomologists or zoologists of the eighteenth century (thus proving that, contrary to a widely distributed opinion, physical 
sciences might follow in their development the biological sciences). Clearly it is no longer possible to be satisfied by this state of affairs. The age of computers has finally come and theoreticians are now able to challenge the analytical or numerical difficulties that one meets when trying to understand nonclassical situations.

In this theoretical approach there are two phases: (a) The first problem to solve, as usual, is that of diagnostics. This is essentially what we have tried to do during these three short days. We have tried to interpret the observed features in terms of models, in the broader sense of this word. I shall come hack to this point.

(b) The second problem is the physical interpretation or understanding of models that fit the observations better. We barely touched this problem and we hardly mentioned the physical choice among the variety of models that can represent a given set of observed features. Clearly this approach is linked with stellar evolution, for stellar evolution might affect more rapidly the nondense external layers than it does the general structure of a star. It is only after we shall be able to enter this second phase of the theoretical approach that we may be able to put some unity, or at least some logic, into the description and understanding of our zoological garden.

I shall come back to this point at the end of these final remarks. In a general sense, we should keep in mind the few questions asked a long time ago by Otto Struve, and of which Anne Underhill has very wisely reminded us. We should remember they are still basically unsolved, and are still the essential questions. Slightly reformulating the first ones, one has to reply to the questions: Why have some stars extended atmospheres--whatever meaning each of us gives to the words--and some other stars, otherwise apparently identical, have none? What, in the stars with extended atmospheres, is the cause of the departures from $R E, H E$, and LTE that one needs to introduce in order to interpret the observations?

It seems that to the last question at least we have a partial reply, which we all seem to agree upon, and about which Anne Underhill and several other speakers have been insisting very much and very rightly. It seems indeed that we do know why there are departures from LTE in a given configuration--or to say it better, why we should not expect LTE to apply. For instance, as stressed by 
Anne Underhill, we know that in low density atmospheres we should not expect enough collisions to maintain the extreme situation of LTE. That this is indeed true for all atmospheres has been stressed by R. N. Thomas. This is clear, and I will not come back to this point.

But why are there departures from RE or HE?

What heating mechanisms can possibly alter the electron temperature aistribution of a classical atmosphere? What is sustaining or pushing the atmosphere in such a way as to produce low effective gravities in the outer layers? We do not know! Our ideas are too vague to allow us to compute these effects in a stellar atmosphere defined by a few observable parameters such as its location in a color-magnitude diagram, or even for à stellar atmosphere much better. defined, such as that of the sun.

One can hope that our improvements in the nonLTE atrophysics will enable us to describe in a better way the observations, one cause of the indeterminacy being thus taken away. Figure 1 describes the logic of the methods in use.

In addition, the kind of wrong and artificial determinations that might have been given to the problem by the use of LTE astrophysics and that might have led in some cases to wrong conclusions about the geometry or the velocities, should be now easily avoided. We know that LTE is an assumption that one cannot make a priori, that one cannot use this assumption unless it has been unambiguously proved in a given particular case, that it may, indeed, be used. We know that this is very rarely the case, especially for stars with extended atmospheres.

How to include non-LTE in the transfer equations and how to do it without neglecting curvature terms or velocity terms, or even inhomogeneities has been discussed here at length and in a very clear way by George Rybicki, and after him by Kalkofen, Magnan, Grant, and Skumanich. However, the theory has still a long way to go. Even in cases with simple velocity fields, we have seen that it is difficult, sometimes, to take into account the proper depth variation of the physical parameters. We have also seen with Nussbaumer that severe doubts still exist concerning the physical data needed for the astrophysical theory. Precise physical data is obviously a serious need of non-LTE astrophysics.

But we have seen also that the theory is sometimes satisfying. The range of possible models not only has decreased but some absurd ones cannot be considered anymore. We have seen that the continuum 
里

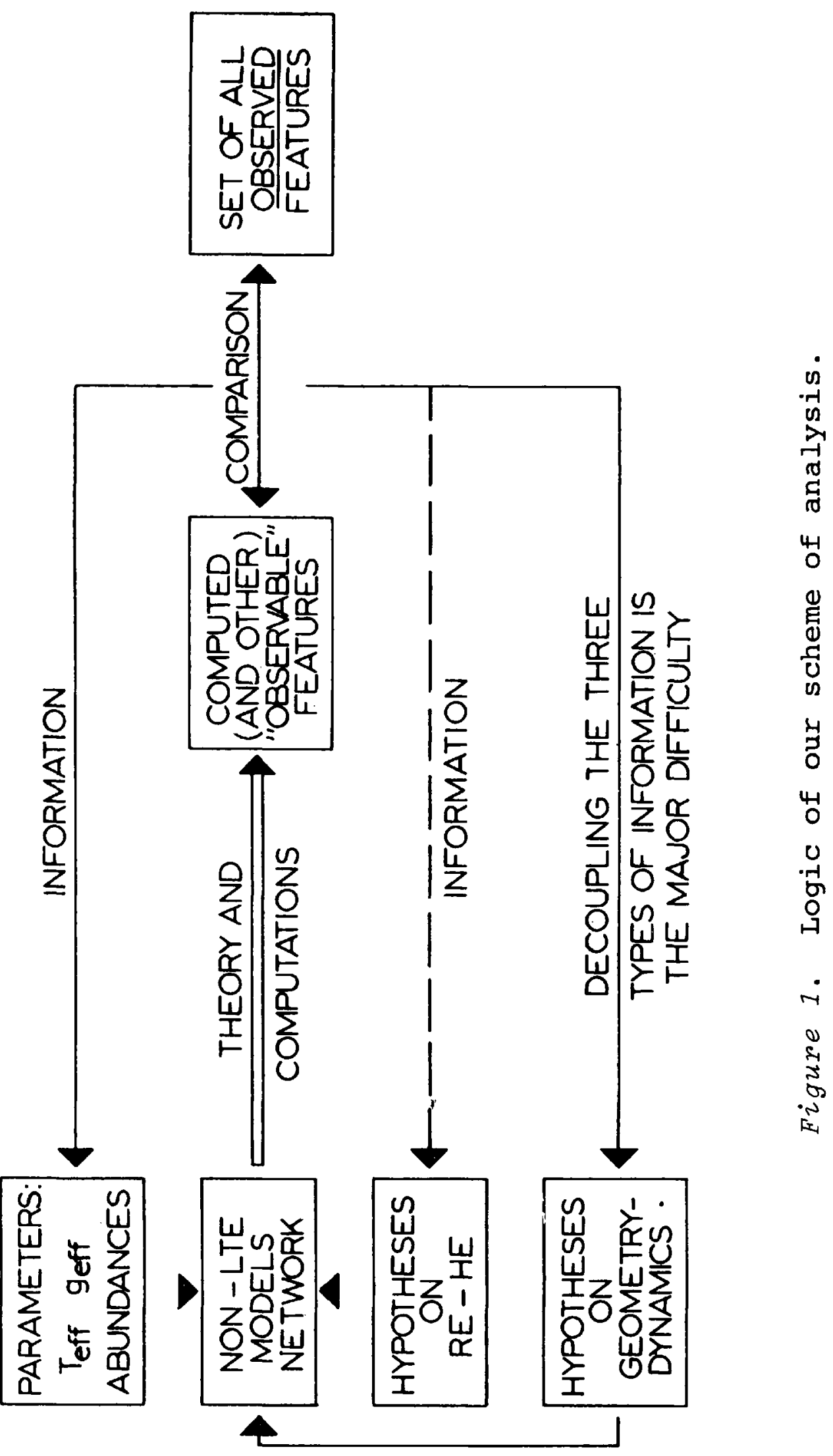


spectrum of a star like Eta Carinae is well represented by the nonclassical continuum proposed by

Gerola. We have seen, in Kalkofen's paper for example, good (but possibly still not unique) representations for $\mathrm{P}$ Cygni lines; Rybicki gave us some examples of profiles of which the asymmetry computed was comparable to those of actual supergiants. Magnan has succeeded in mimicking the Be stars reasonably well; the analysis of WR lines with some non-LTE profiles in plausible geometrical dynamical situations has been noted several times. We have also seen that chromospheric spectral features might be described by some reasonable models, such as those of Kandel, reported this morning by Françoise Praderie. We have seen also the notable progress described by Groth on the chromosphere of the $K$ type component of Zeta Aurigae. Even in the peculiar case of novae, we are coming to a better understanding of the spectrum by making a careful discussion of the ionization, as shown by Wellman.

Certainly even when the crude approximations sometimes made are taken away, a unique description is not yet reached. This has been shown this morning by Groth, but was clear also from several examples given by F. Praderie, and quite clear from

Skumanich's interpretation of the $\mathrm{K}$-line reversal in a few red giants.

Coming to this last controversy, we must understand the indeterminacy. A given line is indeed an image, through some convolution, of the trend of the source function with the optical depth. To go from that law to the distribution of physical quantities, we need a model. The critical relation in it might be $\tau(h)$, the relation between the opacity and the altitude in the atmosphere. Eclipses can lead us to guesses about $\tau(h)$ but in other cases we are left without much indication of this behaviour. The relation $\tau(h)$ is strongly coupled with the set of temperatures or densities as a function of the altitude which are coherent, through non-LTE theory, with the source function itself. This difficulty, I believe, is the chief difficulty we shall meet in the stellar cases, the very origin of the indeterminacy mentioned above. Possibly only careful solution of the simultaneous problem of several different lines can help us. The profile of a line is an indication about $S(\tau)$, but the profile is not dependent on one function only but upon relations such as $T_{e}(h)$ and $n_{e}(h)$. How then to solve for this uncertainty of the diagnostics, an uncertainty which is quite general? 
In given cases we get a set of possible behaviours of $\mathrm{T}_{\mathrm{e}}(\tau), \tau(h)$ and $v(h)$ that depart more or less strongly from the radiative equilibrium temperatures, from the hydrostatic equilibrium relation between $\tau$ and $h$, and from the non-moving atmosphere. The choice is to be determined by the physical plausibility of the models. However we know very little about how to make this choice--as has been said many times during these three days!

\section{APPLICATIONS}

The methodology being thus clear, but still insufficient, let us see if we can describe in some slightly coherent way the various phenomena we have been talking about. We have considered several types of extended atmospheres following more or less Thomas' classification scheme. It is somewhat difficult or even meaningless to locate some of the types of object we are considering on a colormagnitude diagram, but let us attempt it (see Figure 2).

(1) We have talked about stars in which the extended character is rather severe (WR stars, supergiants, Be stars). These stars are generally highly luminous, in rapid evolution, and some of them are relatively young. It is remarkable, as in the case of the nuclei of planetary nebulae, to see that they are located in the HR diagram near the instability limit set up by the radiation-pressure negative gravity. In other words, the physical phenomenon that appears important is essentially a mechanical support of the outer layers. Clearly the difference between objects with and without an extended atmosphere (everything else being alike) might be a difference of age--a difference that can be indeed linked also with really circumstellar phenomena such as dust clouds. The kind of theory brilliantly developed today by Hillendahl could be compared to this case. Through shock waves reaching the surface, a low effective gravity atmosphere is created--even relatively far from the above quoted instability limit.

(2) Later we turned our attention to more moderate examples, for which we suspect that phenomena similar to those shown in an embryonic way in the sun are also present, possibly more conspicuously. This subject was extensively discussed by F. Praderie.

Different mechanisms that act in different ways can be invoked. In the sun and similar stars, the 


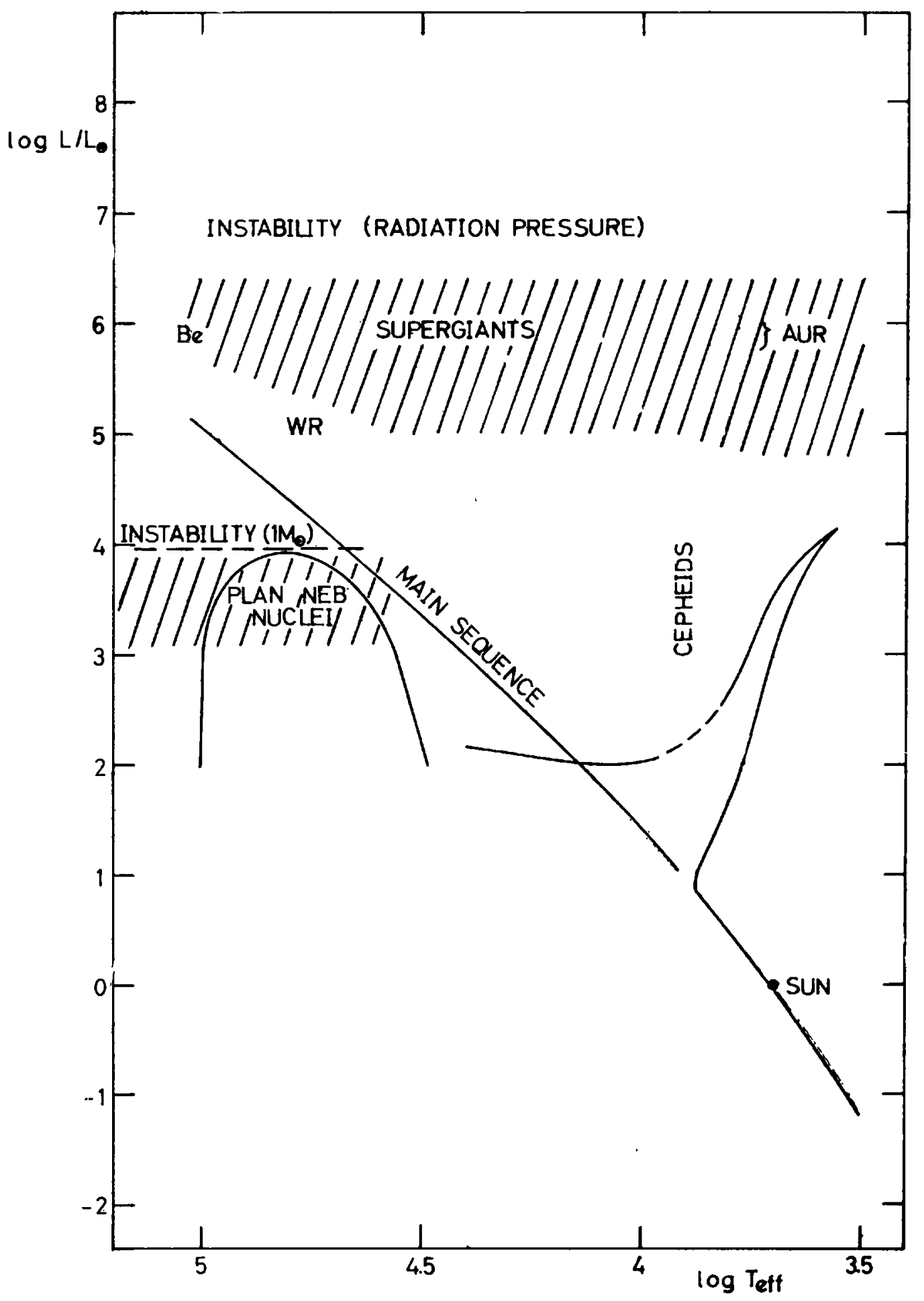

Figure '\%. Location of stars with extended atmospheres in the HR diagram.

heating (due to what?) is probably more important than the mechanical support. But in active younger stars, such as $T$ Tauri, very active phenomena integrated over the stellar disk might appear to give strong chromospheric-like phenomena. The heating there is closely linked with the magnetic properties 
of the stars--as it may also be in the case of some A stars.

Thomas has shown how the study, not of the sun itself, but of the evolution of thinking in the case of the sun could be mimicked, mutatis mutandi, in stellar cases, account being taken of the fact that the phenomena might be physically different or of a different order of magnitude.

I would like at this point to repeat what Thomas said about the fact that the measurement of continua in UV or IR would be very useful to get the location in the atmosphere of the minimum temperature. To his comments I would like to add that the mass of matter that is above (or outside) the location of the minimum temperature is a parameter which may vary within very large limits; and it might be an essential parameter. Having this remark in mind, one can understand why the UV solar spectrum looks like the visible spectrum of some of the monsterstars; just because the mass of the extended atmosphere--or call it chromosphere if you like--is bigger, much bigger, than that of the sun quite irrespective of what the physical process is which keeps supporting the extended atmosphere. This really explains why the discussion of some features of the UV spectrum of the sun (O I lines) by Athay fitted into the program better on Monday together with the discussion of the chromospheric phenomena.

If we limit ourselves to the observation of the visible integrated light of a star, the chromospheric phenomena in objects that might be low in the HR diagram can be observed, as has been told by F. Praderie, only in the center of some very strong lines. This is naturally a severe difficulty.

When dealing with this kind of very fine and difficult spectroscopy one cannot be satisfied with the kind of rough theory that was described yesterday. It became clear today, at several moments in the discussion, that we should improve the theory greatly to take into account inhomogeneities and to introduce wavelength-dependent source functions, i.e., better frequency redistribution in lines than is generally assumed. Clearly a lot of progress has yet to be made along these lines.

\section{CONCLUSION}

Clearly observers should observe and theoreticians theorize, and they should incubate together! 
We now have a better idea which lines are sensitive to what and what theories are likely to be applicable in a particular case. Let us all remember the philosophy of two famous thinkers: "Things are not what they seem" (Thomas) and "I was pleased--and then I started reading" ( $A$. B. Underhill). In no better way can the need for controversy and for colloquia be expressed.

\section{ACKNOWLERGMENTS}

Yesterday, when Dr. Wellmann was replying to the expression of our.gratitude that had been given by Anne Underhill, he said that it might be too early, the meeting being not yet over. I may say today that, the meeting being over, we know perfectly well that Anne Underhill was completely right; it is a great pleasure for me to again thank very heartily Dr. Wellmann and Dr. Groth for all that they have done in order to allow us to take the utmost benefit out these three days, not only through scientific discussions, but through our contacts with our German friends. I would like, Dr. Wellmann, to ask you, also, to convey our thanks to the secretarial staff, which has been so helpful for all of us, and also to your colleagues and students who have been helping so much in many ways, and especially by recording the whole of this conference, and by taking accurate notes during it.

It is customary to limit the thanks to the local organisers, but I think we should also express our gratitude to Anne Underhill, who has been so active in organizing this meeting, and who, fortunately, let it be sufficiently unplanned. It was indeed an excellent thing that this meeting was unplanned, because it gave us the opportunity of extensive discussions about the various points raised by the speakers. I thank also all those who have contributed to the discussion, especially (for different reasons) the younger ones, and our old friend Dick Thomas.

I have said earlier that we are able, now, to express our gratitude to Dr. Wellmann and Dr. Groth, the meeting being finished. Actually, we should possibly wait a little bit, because they have still an enormous amount of work to do with the proceedings of the conference! This is an enormous task, but judging by what I have seen these days, I think it 
is quite safe that we can express our gratitude in advance to Dr. Wellmann, Dr. Groth, and their colleagues, for what they will do for us in the near future.

The meeting is adjourned. 